\title{
Analysis of Marketing Management and Process Optimization based on Big Data
}

\author{
Lan Wu \\ Chongqing Vocational Institute of Engineering, Chongqing, 402260, China
}

\author{
Keywords: Market Management, Big Data, Process Optimization
}

\begin{abstract}
This paper utilizes and integrate effective information, discovers valuable data, uses big data to drive companies for precise marketing, and provides decision support for corporate marketing management. Big data has changed the company's cognitive model of marketing management. With the continuous development of big data technology, it will inevitably lead to innovation and change in corporate marketing management. Applying big data to marketing management and promoting innovation in marketing management based on big data background can help enterprises improve marketing strategies, further tap potential customers, and enhance marketing effects, thus achieving double improvement in corporate brand influence and economic efficiency.
\end{abstract}

\section{Introduction}

Big data has profoundly affected many fields such as economy, society, education, etc. Whoever has big data has a future. The use of big data to form consumer behavior analysis is the basis for enterprises to develop marketing strategies. The calculation and application of big data can help enterprises collect and analyze the online data of consumers, and enterprises can make reasonable decisions in marketing management as soon as possible. Big data has changed the company's data analysis thinking and has an irreplaceable role in helping companies adjust their marketing strategies. For enterprise marketing management, big data impacts the company's marketing management system and increasingly presents a business value that cannot be ignored. As an important resource, big data has penetrated into various industries in different degrees. Applying big data to enterprise marketing management not only helps enterprises to operate, but also promotes the development of national economy. Therefore, enterprises must Develop a big data marketing strategy plan in advance to seize market opportunities. To carry out big data marketing, enterprises must carry out marketing innovation and reconstruct the marketing system under the era of big data. Collecting and arranging user information and effectively calculating and processing customer data can help enterprises adjust marketing strategies in a timely and effective manner and achieve precise marketing.

\section{Application of big data in management marketing}

Using the historical data generated by consumers when browsing and purchasing goods, the consumers' personal preferences and needs are inferred, and the future shopping behaviors and needs of consumers are further predicted, and the corresponding product information is accurately pushed to the consumption. In front of the people, maximize market opportunities. For example, a classic case in the United States is about a 16-year-old girl who received a promotional coupon for a maternity store, which made her father angry to find a mall to find justice, but did not expect her daughter to be pregnant. The reason is that the mall based on big data analysis, predicting the possibility of a girl's pregnancy within a small margin of error, thus preempting the market opportunity.

Customer-centric, using big data technology to drive and provide personalized services for consumers is an important value generated by big data in marketing management applications. In order to make products more close to the needs of consumers, enterprises need to use big data to 
judge consumer preferences, as well as requirements for product quality and performance, and to carry out targeted product production, thereby increasing product sales. Based on big data analysis, in the consumer's research on household cars, consumers show individual differences in terms of brand, appearance, fuel consumption, price, and safety factor preference. One type of consumer requires relatively high prices and comfort levels for cars, while another type of consumer requires relatively high prices and safety factors. To this end, according to the degree of consumer preference, the car can be subdivided into "comfortable configuration" and "safe and economical". The comfort configuration is designed and manufactured to emphasize the comfort of the vehicle. The safety and economy are designed and manufactured to emphasize the safety and fuel consumption of the vehicle.

Thousands of products make it difficult to implement time-consuming and labor-intensive manual price settings, so that complex pricing variables cannot be properly processed and refined. The use of big data allows companies to more intuitively capture price influencing factors and make pricing more reasonable. Enterprises must set the most reasonable price, not only have a huge database, but have strong analytical capabilities. The big data automatic analysis system can refine the customer base and accurately analyze the influencing factors of the transaction efficiency of each customer group. On this basis, the company can accurately set the targeted price for the corresponding customer group. At present, many companies have made significant decisions using pricing decisions made by big data. A European building materials company used big data pricing strategies to increase product profits by $20 \%$. If companies want to set the right price, they should make full use of big data, otherwise it means a large loss of profits.

\section{Problems and shortcomings in big data marketing management}

Now, users want to experience convenience through big data. For enterprises, how to extract the parts that can be effectively utilized from the massive data, and use them for enterprise marketing management is the magic weapon for enterprises to win. The Internet era generates data all the time, extracting and analyzing massive data information, provided that the information must be true and objective feedback from users. For the difference in effective information collection and identification capabilities, big data is vulnerable to invalid or invalid information, and the reliability of analysis conclusions is biased. For example, the massive derivation of the network water army has greatly affected the authenticity and effectiveness of information data. Taobao sellers brushed the credibility, brushed sales, brushed praise, so as to get the top ranking, so that the goods can be displayed first, in order to increase sales, resulting in unfair competition between the stores. Massive data is exploding every moment, collecting and extracting relevant data in massive data, and finally drawing conclusions through various calculations, which makes it difficult for companies to keep up with the pace of data growth and change. For example, the data platform predicts that a movie will have a high box office, but the result will be dismal. As for those who claim to use big data to predict the results of the game and win the election, they are often miscalculated. Big data is only extracted from historical data and analyzed and the results are obtained. However, market changes are unpredictable. In marketing management, decision makers need to refer to big data and combine current market development changes instead of blindly following big data. Analyze the conclusions and make corresponding adjustments to the marketing management of the enterprise. Otherwise, the adjustment of the marketing management strategy of the enterprise cannot meet the market situation.

In the era of big data, when sorting out large amounts of information, it is necessary to extract a large amount of user information, and the resulting privacy and security issues have exploded. Since a series of data generated by the user's Internet access contains some identification information, such as an account name, a password, and the like, the cached data generated by the user's computer operation may be stolen or tampered with during a malicious attack. For enterprises, although they can increase the accuracy of their advertising, they also face the risk of malicious programs stealing corporate information. In June 2015, ICBC's fast payment was exposed to serious loopholes, and many depositors in the Beijing area suffered from theft of deposits. Criminals intercepted deposit 
card deposits by illegally intercepting SMS verification codes. In August 2015, the US marital affairs website "Ashley Madison" was hacked and published details of the members of the website on the Internet, saying that the move was to force the website to stop. Leading users and websites to serious attacks from all sides.

\section{Improve the improvement suggestions of big data in marketing management application}

The use of big data to enhance corporate marketing management is to adapt to the development trend of the information age, is the urgent need to provide personalized services to customers and comprehensively promote marketing management concepts. Whether the data is garbage or baby depends on whether the company has the ability to understand big data and mine and use data in marketing management. At present, the penetration of global big data into various industries has become a development trend. If the "ostrich policy" is adopted, the result can only be eliminated by the era of big data. Enterprises must apply big data to marketing management, establish big data awareness at the enterprise level, and form a corporate big data culture. Building a big data culture is to form a data-based value and institutional system throughout the enterprise that provides the foundation for companies to truly leverage big data to generate value. Enterprise data culture is mainly reflected in the implementation and management of enterprise marketing, and its decision-making must be conducted through data.

Dr. Ren Yongjie, president of the domestic database enterprise Renmin Jincang, said bluntly: "Under the big data trend, the related applications around the value of data are complex and changeable. Whether it is a database vendor or a customer, it is necessary to change the role orientation, take the data value as the core and step into the consciousness. , technology, products, services and the application of a large service era." Enterprises want to make marketing decisions and customer service, you need to do research on the market situation, but the information in the research process may not be suitable for business needs. Because under conscious conditions, answering questions is somewhat insincere. But big data is different. It is the real information feedback made when the customer is not aware of being supervised. If the data can be mined and relied on big data technology, the customer's consumption behavior is analyzed in detail, and the customer group is accurately classified. To establish a comprehensive enterprise and customer interaction system, we will be able to achieve refined marketing management and maximize benefits. Before applying big data marketing management, enterprises mainly rely on financial data for market analysis and marketing management decisions, while big data is to use all enterprise related data (such as customer feedback, competitor information, etc.) to guide the company's marketing management strategy and decision-making. effect. Enterprises want to apply big data in marketing management. What they need to do is not to follow the crowd, but to learn how to use massive data. Big data technology is an innovative thinking for corporate marketing management. Many companies always think about how to back up and process historical data, rather than for analysis and research, so that the data is only for the purpose of checking, but can not play the role of data in marketing management applications. At the same time, many companies use big data only to focus on internal data, while ignoring the effectiveness and value of massive external data. At the same time, big data is not omnipotent. Enterprises can't rely too much on big data. Big data is based on the algorithm and screening ability of massive information, which leads to the deviation of the true validity of data. Therefore, enterprises should try in the process of marketing management application. Do some analysis and verification.

\section{Conclusion}

Utilize and integrate effective information, discover valuable data, use big data to drive enterprises to conduct precise marketing, and provide decision support for enterprise marketing management. Big data has changed the cognitive mode of marketing management, with the continuous improvement of big data technology. Development will inevitably lead to innovation and change in corporate marketing management. 


\section{Acknowledgements}

Fund Project: Chongqing Social Science Project (Provincial Level) 17SKG277: Research on the Impact of Social Network on Residents' Income Gap Economics

Moderator: Wu Lan

Chongqing Engineering Vocational and Technical College Planning Project

\section{References}

[1] Huang Shengmin, Liu Shan. Deconstruction and Reconstruction of Marketing System under the Background of "Big Data” [J]. Modern Communication, 2012(11).

[2] He Jun. Analysis of the Impact of Big Data on Enterprise Decision-making [J]. Science and Technology Progress and Countermeasures, 2013(9).

[3] Kong Weicheng. “Network Marketing” [M]. Beijing: Higher Education Publishing House, 2005.

[4] $\mathrm{Hu}$ Xiaoming. Misunderstandings, risks and advantages of big data applications [J]. E-Government, 2014 (11).

[5] Meng Xiaofeng. Big Data Management: Concepts, Technologies and Challenges [J]. Computer Research and Development, 2013(1) 